\title{
APAPARI Educational Lecture1
}

\section{From birth to adolescence-the atopic march}

\author{
Ulrich Wahn
}

Department for Pediatric Pneumology and Allergology, Charité, Berlin, Germany

The term "atopic march" refers to the dynamic process of manifestation, persistence and remission of different atopic phenotypes in the first two decades of life. In general the first manifestation of atopy is infantile eczema which manifests between the second and sixth months of life and may persist for a couple of years. The majority of infants with atopic dermatitis will develop partial or complete remission by school age.

During the first months of life a subgroup of atopic dermatitis children will develop a sensitisation and allergy to food proteins like hen's egg, cow's milk, soy or peanut. The majority of infantile food allergy is likely to go into remission after a couple of years. The serum concentration of specific IgE-antibody concentrations to food proteins has been shown to be a determinant of the time of persistence of infantile food allergy.

Between the third and tenth year of life allergic manifestations of the upper or lower airways develop which may persist into adulthood. Allergic rhinitis can be considered a prodromal state of asthma. Persistant asthma is more likely in children with early sensitization to indoor allergens (dust mites, cats) and high infantile domestic allergen exposure.

In association with pollen allergy a significant percentage of children will develop pollen associated food allergy due to panallergens which are responsible for cross-reactivity between different food sources. 


\title{
APAPARI Educational Lecture2
}

\section{Allergy in School (EAACI task force)}

\author{
Magnus Borres \\ Department of Pediatrics, Faculty of Medicine Gothenburg University, Sweden
}

Children with a high risk of having asthma attack or anaphylactic reaction should receive special attention at school. Schools also have a unique opportunity to detect and recognize children with allergic symptoms. An early diagnosis is important for the allergic children (1) because it influences the prognosis of the disease and also prevent learning difficulties.

The aim of this Task Force initiated by EAACI and the European Galen network was to describe an ideal model of care centered on the allergic children at school. Despite a substantial body of literature on allergy management, the evidence available on how to best care for such children at school is inadequate. Furthermore, legislation, education, facilities and health care practices vary between countries. The Task Force has presented the information in such a way that individuals will be able to adopt the advice within the context of their local or national facilities to improve care for all children with allergy at school (2). Where local facilities are not able to support such an approach, this document may be used as a reference to inform national policy.

This position paper is the first document aiming at standardize management and increase awareness for these children. Some countries like Sweden had national guidelines for managing the schoolchildren with asthma and allergy (3) and some countries like France had to some extent appropriate legislation in order to ensure a safe school environment for this group. The majority of European countries had no guidelines or legislation in this area before. The work now consists of implementing this document in the different European countries.

The focus on this talk will the Position Paper stating that recognition of the allergic child is the first step in management. Schools should enquire about a diagnosis of allergy at the registration of any new child. There should be protocols in place to ensure rapid access to emergency medical care. The school may hold treatment protocols and allergy medication for emergency use, where allowed by national laws.

Further, an education network involving families, healthcare and education providers will be discussed as crucial in ensuring that children are identified, the school staff alerted and trained, and specific allergy management plans initiated. This should be achieved through the empowerment of key stakeholders and supported by continuing education of all school staff.

Appropriate legislation should be introduced to make safe the school environment for the allergic child as well as safeguarding educational workers. The implementation of these recommendations across diverse models of healthcare in Europe would ultimately protect and nurture all children with allergy whilst at school.

(1) Allergy testing in children : Why, Who, When and How? Höst A et al. Allergy 2003 ; 58 : $1-11$.

(2) The management of the allergic child at school : EAACI/GA2LEN Task Force on the allergic child at school. Muraro A et al, Allergy. $2010 ; 65: 681-9$

(3) Asthma and allergies at school-a Swedish national position paper. Borres MP et al. Allergy $2002 ; 57: 454-457$ 


\title{
APAPARI Educational Lecture3
}

\section{Re-evaluation of "Hygiene Hypothesis"}

\author{
Kenji Matsumoto \\ Department of Allergy and Immunology, \\ National Research Institute for Child Health and Development, Japan
}

Frequent upper respiratory infections and chronic exposure to environmental endotoxins during early life protect infants from subsequent sensitization to airborne allergens and from development of asthma and allergic rhinitis. This phenomenon has been called the "Hygiene Hypothesis." The microbial stimuli are thought to shift "airborne antigen"-specific immune responses towards Th1 by activating the innate immune system on dendritic cells. Thus, the effects of unhygienic conditions have virtually no involvement in the development of atopic dermatitis or food allergy.

On the other hand, individuals with impaired local innate immune responses to common cold viruses, including respiratory syncytial virus and rhinovirus, or those with impaired local responses to bacterial pathogens, often develop lower respiratory infections with wheeze, or skin surface infections, upon exposure to weakly virulent pathogens. These individuals are thought to be at high risk of subsequent asthma or atopic dermatitis even though they have frequent upper/lower respiratory infections, because lower respiratory infections or skin infections damage epithelial barrier functions and increase organ-specific hypersensitivities that may lead to the development of allergic diseases.

Therefore, innate immune systems play critical roles in both induction of airborne antigenspecific Th1 immune responses and local protection against weakly virulent pathogens. Moreover, it is likely that interactions between environmental microbial exposure and the genetic predispositions of the receptors of microbes or the molecules involved in signal transduction pathways regulate the onset of allergic diseases. The development of strategies for prevention of allergic diseases should take such gene-environment interactions into account. 


\title{
APAPARI Educational Lecture4
}

\section{Adipokines Affect Exercise-induced Bronchoconstriction in Childhood Asthma}

\author{
Ha-Baik Lee, MD \\ Department of Pediatrics, Hanyang University School of Medicine, Seoul, Korea
}

Recent epidemiological studies have shown that increasing prevalence of asthma and obesity is emerging as major health problem. Moreover, exercise-induced bronchoconstriction (EIB) frequently affects daily activities as well as school performance in children. However, the pathogenesis and proper treatment modality of EIB in asthma is still controversial. It is known that exercise causes bronchoconstriction because of water loss by evaporation from the surface of the airways resulting in increased airway osmolarity and mast cell activation.

On the other hand, obesity is a risk factor for bronchial hyperesponsiveness (BHR) to methacholine with increasing BMI in obese asthmatic children. Generally EIB was significantly higher in obese asthmatics than in non-obese asthmatics. Because exercise challenge causes airway smooth muscle contraction through one or more intermediate mechanisms, such as neuronal reflexes and release of inflammatory mediators by inflammatory cells. Recently, human mast cell expresses leptin receptors (Taildman 2009), which suggests mast cell activation is feature of EIB (O’Sullivan S. et al. 1998). To date, adipokine-related effects on EIB have not yet been sufficient for confirmation in the human airway.

We investigated the effect of leptin and adiponectin on EIB development in overweight asthmatic children. Results have shown that overweight EIB denoted lower $\mathrm{FEV}_{1} / \mathrm{FVC}$ ratio and less post-BD improvement of $\% \mathrm{FEV}_{1}$. Serum adiponectin and leptin were lower and higher, respectively, in obese children with asthma than in normal-weight children with asthma, and were significantly correlated with BHR induced by exercise challenge. Also, leptin/adiponectin ratio was correlated with $\mathrm{BMI}(\mathrm{r}=0.445, \mathrm{p}<0.05)$. When we examined the relationship between exhaled nitric oxide (eNO) and childhood EIB, baseline eNO levels correlate with the post-exercise decrease of $\mathrm{FEV}_{1}$, suggesting that eNO may be a tool in prediction of EIB in noninvasive manner.

To verify whether mast cell activation is a feature of exercise-induced bronchoconstriction or not, we studied relationship between leptin and adiponectin and EIB by measuring urinary metabolites of mast cell mediators. From the results, the adipocyte-derived hormones leptin and adiponectin were correlated with BHR and urinary metabolites of mast cell mediators induced by exercise challenge in asthmatic children (Baek et al. 2011).

Finally, we conducted another study to find proper treatment of EIB by comparing the pathobiologic effect of oral leukotriene receptor antagonist (LTRA, montelukast) and an in- 
haled glucocorticoid (budesonide) on EIB therapy in asthmatic children. There was overproduction of $\mathrm{LTE}_{4}$ as distinctive immunopathologic features of asthma with EIB. So LTRA therapy was more effective in improving EIB than an inhaled glucocorticoid.

In conclusion, our study results suggest that adiponectin and leptin might involve in the development of EIB via mast cell activation in obese asthmatic children. Also LTRA was equivalent to an inhaled glucocorticoid or more effective as controller therapy for EIB in children.

\section{References}

1. O'Sullivan S, Roquet A, Dahlen B, et al. Evidence for mast cell activation during exerciseinduced bronchoconstriction. Eur Respir J 1998; $12: 345-50$

2. Shore SA. Obesity and asthma : possible mechanisms. J Allergy Clin Immunol 2008; 121 : 1087-93; quiz 94-5.

3. Shore SA, Schwartzman IN, Mellema MS, Flynt L, Imrich A, Johnston RA. Effect of leptin on allergic airway responses in mice. J Allergy Clin Immunol $2005 ; 115$ : 103-9.

4. Taildeman J, Perez-Novo CA, Rottiers I, et al. Human mast cells express leptin and leptin receptors. Histochem Cell Biol 2009 ; 131 : 703-11.

5. O'Sullivan S, Roquet A, Dahlen B, et al. Evidence for mast cell activation during exerciseinduced bronchoconstriction. Eur Respir J $1998 ; 12$ : 345-50.

6. Baek HS, Kim YD, Shin JH, Kim JW, Oh JW, Lee HB. Serum leptin and adiponectin levels correlate exercise-induced bronchoconstriction in children with asthma. Ann Allergy Asthma Immunol 2011 (in press)

7. Price D, Musgrave SD, Shepstone L, Hillyer EV, Sims EJ, et al. Leukotriene Antagonists as First-Line or Add-on Asthma-Controller Therapy. N Engl J Med 2011 ; 364 : 1695-707 


\title{
APAPARI Educational Lecture5
}

\section{Gastroenterology and Allergy}

\author{
Ralf G Heine MD FRACP \\ Royal Children’s Hospital, University of Melbourne, Australia
}

Food protein-induced gastrointestinal syndromes are relatively common in infants and young children. While cow's milk allergy accounts for the majority of cases, other food proteins are commonly involved (e.g. soy, wheat). Gastrointestinal food allergy may present with a diverse range of clinical manifestations. In addition to the 'classic' clinical manifestations of gastrointestinal food allergy (i.e., food protein-induced enteropathy, proctocolitis and enterocolitis syndrome), there is increasing evidence that food proteins may be of importance in the pathophysiology of other common paediatric gastrointestinal disorders (i.e., gastro-oesophageal reflux disease, infantile colic and constipation). Eosinophilic oesophagitis is a newly emerging allergic manifestation which is closely associated with $\operatorname{IgE}$-mediated and non-IgE-mediated food allergy. These gastrointestinal food protein-induced diseases still pose diagnostic and therapeutic dilemmas due to a lack of clear diagnostic markers and clinical overlap with other diagnoses, including lactose intolerance, coeliac disease or gastroenteritis. The present lecture will discuss pathways for the diagnosis and treatment of gastrointestinal food allergy, with particular focus on the pathophysiology and differential diagnosis. 


\title{
APAPARI Educational Lecture6
}

\section{New insights into immune development and allergic disease}

\author{
Susan Prescott \\ University of Western Australia School of Paediatrics and Child Health, \\ Princess Margaret Hospital, Perth, Australia
}

Allergic and nonallergic children show dramatic differences in the patterns and trajectory of their immune development. This includes well-documented developmental differences in $\mathrm{T}$ cell function, innate cell function as well as more recently recognized emerging difference in regulatory $\mathrm{T}$ cell (Treg) function.

We have previously shown that nonallergic children show a progressive age-related maturation in Th1 IFN $\gamma$ responses during the postnatal period, with suppression of the normal perinatal predisposition for Th2 immunity. We have also shown that this is associated with parallel maturation in innate immune function, characterized by progressive and significant age-related increases in innate cytokine responses ( $\mathrm{IL}-1 \beta, \mathrm{IL}-6, \mathrm{TNF} \alpha \mathrm{IL}-10)$ to virtually all microbial toll-like receptor (TLR) ligands. More recently, we have shown that developmental changes in the thymus parallel recognized changes in peripheral blood responses, and that thymic-derived CD4 + CD25 + CD127lo/-FOXP3 + Treg expansion and suppressive function increase with age and paralleled increase in global thymic FOXP3 mRNA expression in nonatopic children.

In clear contrast, allergic children show a characteristic age-dependent increase in allergenspecific effector Th2 responses with attenuated maturation of Th1 IFN $\gamma$ function. These children show exaggerated innate responses at birth but a relative decline with age thereafter, so that by 5 years of age responses are attenuated compared with nonallergic children. Thus, this early hyper-responsiveness in allergic children fails to translate to a corresponding maturation of Th1 function, which remains attenuated relative to nonallergic children. We have also recently shown developmental delay in function of thymic Treg in atopic children with significantly impaired maturation compared to age-matched nonatopic children.

Our findings indicate significant differences in the developmental trajectory of immune function in children who develop allergic disease that may collectively contribute to disease predisposition. Dysregulated inflammatory responses may have a role in abnormal T cell development, and that impaired regulatory function may also have a role in failing to regulate these aberrant responses.

Notably, differences in these and other aspects of immune function are already emerging at birth, highlighting that environmental effects are begin during intrauterine life. We speculate that, differences in early immune function seen in allergic and nonallergic infants reflect combined effects of maternal phenotype, infant genotype and environmental exposures in utero on early gene expression. 


\title{
APAPARI Educational Lecture7
}

\section{Viruses and Asthma}

\author{
Joon Sung Lee \\ Seoul St. Mary’s Hospital, the Catholic University of Korea, Korea
}

Respiratory viral infections can cause wheezing illnesses in children of all ages and possibly influence the development and severity of asthma. Respiratory viruses have been shown to be epidemiologically associated with asthma in several ways. First, certain viruses associated with infantile wheezing have been suggested to be responsible for the inception of the asthmatic phenotype. Second, in children with established asthma, viral respiratory tract infections have been shown to play a significant role in producing acute exacerbations that may require healthcare services for management.

Despite the abundance of studies addressing the role of viral infections in the development of asthma, definitive conclusions cannot yet be draw about the causal nature of the relationship. From the available evidence, the development of asthma emerges from a complex interaction of genetic predisposition and environmental factors with viral infection likely playing a significant role in the effect of environment on asthma inception. Continued evaluation of this interaction with epidemiologic investigation, cohort studies, animal models, and in vitro evaluation is required to gain new insights and answers to these issues.

Treatment of virus-induced wheezing and exacerbations of asthma can be challenging, and several studies have evaluated the current treatment strategies. Current studies aim at determining the mechanisms by which viruses may cause acute wheezing and more importantly, the numerous ways in which severe viral infections influence the pathophysiology of asthma in numerous ways. As soon as these mechanisms are established, a more definite identification of children who are at the greatest risk for wheezing with viral infections or in whom the existing virus-induced wheezing preludes asthma may be possible. Several host factors, including respiratory allergy and virus-induced interferon responses, modify the risk of virus-induced wheezing.

The other obvious rationale for identifying pathogenic mechanisms of virus-induced wheezing is the identification of targets for novel therapeutic strategies. The current standard therapy for asthma is unsatisfactory because in cases of respiratory infections, its efficacy is low, and in cases of systemic corticosteroids administration, this therapy can cause significant adverse effects. Since viral infections in children are closely related to wheezing illnesses, antiviral strategies should be developed for the prevention and treatment of asthma, and both human rhinovirus and respiratory syncytial virus should be targeted for this purpose. 